\title{
Modified citrus pectin inhibits galectin-3 function to reduce atherosclerotic lesions in apoE-deficient mice
}

\author{
YONGGANG LU ${ }^{1}$, MINGMING ZHANG ${ }^{1}$, PEI ZHAO ${ }^{1}$, MIN JIA ${ }^{1}$, \\ BING LIU $^{2}$, QIAN JIA ${ }^{1}$, JUN GUO $^{3}$, LIN DOU ${ }^{3}$ and JIAN LI ${ }^{3}$ \\ ${ }^{1}$ Clinical Laboratory, Hebei General Hospital, Shijiazhuang, Hebei 050051; ${ }^{2}$ Neurology Department 2, \\ Handan Central Hospital, Handan, Hebei 056001; ${ }^{3}$ Key Laboratory of Geriatrics, Beijing Institute \\ of Geriatrics, Beijing Hospital, Ministry of Health, Beijing 100730, P.R. China
}

Received January 11, 2016; Accepted January 27, 2017

DOI: $10.3892 / \mathrm{mmr} .2017 .6646$

\begin{abstract}
Galectin-3 is a carbohydrate-binding lectin, which has been implicated in the modulation of atherosclerotic pathophysiology, and is highly expressed in monocytes, macrophages and endothelial cells within atherosclerotic plaques. Modified citrus pectin (MCP) is produced from citrus pectin via $\mathrm{pH}$ and temperature modifications, which break it into shorter, non-branched, galactose-rich carbohydrate chains. MCP is able to tightly bind with galectin-3, via recognition of its carbohydrate recognition domain, and facilitates the modulation of galectin-3-induced bioactivity. The present study explored the effects of MCP on the initiation of atherosclerosis. Eight-week-old apolipoprotein E-deficient mice were treated with $1 \% \mathrm{MCP}$ and fed an atherogenic diet for 4 weeks. The effects of MCP on atherosclerotic initiation were determined by pathological analysis and scanning electron microscope (SEM) imaging. MCP treatment reduced the size of atherosclerotic lesion areas, which was accompanied by decreased numbers of macrophages and smooth muscle cells (SMCs). Furthermore, SEM examination of the surface of the atheroma-prone vessel wall indicated that MCP treatment reduced endothelial injury. To analyze the effects of MCP on monocyte adhesion, firstly, oxidized-low density lipoprotein and various concentrations of MCP $(0.025,0.05$, 0.1 and $0.25 \%$ ) were incubated with the human umbilical vein endothelial cells (HUVECs) for stimulation and following this, the U937 cells were plated onto the HUVECs. The results revealed that MCP reduced the adhesion of U937 monocytes to HUVECs, indicating the adhesion-inhibiting effects of
\end{abstract}

Correspondence to: Professor Jian Li, Key Laboratory of Geriatrics, Beijing Institute of Geriatrics, Beijing Hospital, Ministry of Health, 1 Dahua Road, Dongdan, Beijing 100730, P.R. China

E-mail: lijian@bjhmoh.cn

Key words: modified citrus pectin, atherosclerosis, galectin-3, macrophages
MCP. In conclusion, the present study revealed that MCP, a galectin-3 inhibitor, reduced the size of atherosclerotic lesions by inhibiting the adhesion of leucocytes to endothelial cells. Inhibition of galectin-3 function may be a therapeutic strategy for the treatment of atherosclerosis.

\section{Introduction}

Atherosclerosis is the predominant pathological cause of coronary artery disease, stroke and peripheral vascular disease. Endothelial dysfunction is considered to be the primary step of atherosclerosis, and various stimuli may induce this (1). Synthesis of adhesion molecules and chemokines by dysfunctional endothelial cells results in their cellular junctions becoming leakier; these steps induce early atherosclerotic lesions by mediating transient/rolling-type and firm adhesions of leucocytes and macrophages, as well as facilitating monocyte infiltration into the injured atheroma area (2). Leucocyte adhesion to vascular endothelial cells is a critical step in transendothelial migration. The infiltration of monocytes and other immune cells such as neutrophils, is mainly governed by interactions between chemokines or adhesion molecules and their associated receptors, such as vascular cell adhesion molecule-1 (VCAM-1) to $\alpha 4 \beta 1$ integrin, intercellular adhesion molecule-1 (ICAM-1) to $\alpha \mathrm{L} \beta 2$-integrin, and galectin-3 interaction with integrin $\beta 1, \beta 3$ and VCAM-1, either directly or indirectly (3).

Galectin-3 has numerous cellular locations, including the nucleus, the cell surface, and the extracellular environment. The location of galectin-3 depends on its ability to recognize extracellular matrix (ECM) components; particularly laminin, elastin and fibronectin, and these interactions facilitate cell-ECM attachment and transendothelial migration. Furthermore, this lectin may bridge neutrophilic and monocytic connections with the endothelium, either directly or indirectly (4). The release of reactive species and proteolytic enzymes, stimulated by the adhesion of neutrophils and monocytes, may subsequently induce endothelial erosion, aggravate endothelial cell dysfunction, and result in amplified vascular permeability and leucocyte accumulation in the atherosclerotic area. The predominant adhesion molecules expressed on the surface of neutrophils are $\beta 2$-integrins; these serve a 
critical role in the recruitment and transmigration of neutrophils and monocytes (5-7). This is followed by endothelial cell surface-galectin-3 binding, which strengthens the adhesion (4). Galectin-3 has also been considered to promote cell-cell adhesion through a cross-linking of the Mac-2 binding protein, which is a member of the macrophage scavenger receptor cysteine-rich domain superfamily $(8,9)$.

Galactin-3 may also have a similar effect to monocyte chemoattractant protein-1 (10), whereby extracellular galectin-3 may initiate alternative macrophage activation via interaction with cluster of differentiation (CD) 98 on macrophages. Furthermore, galectin-3 may serve as an advanced glycosylation end product clearance receptor, and a mediator of macrophage-led endocytosis of modified low-density lipoprotein (LDL), thus facilitating foam cell formation and subsequent amplification of inflammation $(11,12)$. Cell surface and extracellular galectin-3 may therefore accelerate or decelerate atherosclerotic initiation and progression, by inducing the accumulation and activation of leucocytes.

Modified citrus pectin (MCP) is a complex polysaccharide obtained from the peel and pulp of citrus fruits. MCP powder is produced from citrus pectin using $\mathrm{pH}$ - and temperature-based processes to break it into shorter, non-branched, galactose-rich carbohydrate chains. This shorter polysaccharide enables MCP to access and bind tightly to galectin- 3 by adhering to the carbohydrate recognition domain, thereby modulating the bioactivity of galectin-3 (13). Previous research indicated that either galectin-3 knockout or oral administration with MCP may reduce the atherosclerotic area in apolipoprotein E (apoE)-deficient mice; however, the exact mechanism remains unclear (14-16). The present study explored the effects of MCP on the initiation of atherosclerosis, and it was demonstrated that MCP may reduce the size of the atherosclerotic lesion by inhibiting leucocyte adhesion to endothelial cells.

\section{Materials and methods}

Preparation of animals. Male apoE $\mathrm{E}^{-/-}$mice (age, 8 weeks, $20 \pm 2 \mathrm{~g}$ ) bred from a C57BL/6J background were obtained from Jackson Laboratory (Peking University Health Science Center, Beijing, China). Mice $(n=16)$ were fed a high-fat, cholesterol-rich atherogenic diet containing $21 \%$ fat, $19.5 \%$ casein and $1.25 \%$ cholesterol for 4 weeks and during the course of the experiment, all mice were allowed ad libitum access to food and water. They were maintained at $20-24^{\circ} \mathrm{C}$ with $45-55 \%$ humidity with a 12 -h light-dark cycle. The mice were divided into two groups: MCP $(n=8)$ and model $(n=8)$ groups. A total of 1\% MCP (Centrax International Corporation, San Francisco, USA) was added into the drinking water of the mice in the MCP group, for 4 weeks. All animal protocols were approved by the Animal Ethics Committee of the Beijing Institute of Geriatrics (Beijing, China).

Quantification of atherosclerotic lesions in the aortic root. Mice were sacrificed and hearts were sectioned throughout the aortic root; serial paraffinic sections of the heart were taken every $10 \mu \mathrm{m}$, according to the identified methods (12). Aortic roots were dissected and fixed overnight in $4 \%$ polymerized formaldehyde, paraffin embedded, and subsequently sectioned into $5 \mu \mathrm{m}$ slices. Every sixth section was stained with a modified Movat pentachrome stain (SS0236; Xinhua Luyuan Science and Technology Ltd, Beijing, China), Sirius Red (DC0041; Leagene Biotechnology Co., Ltd., Beijing, China), to evaluate plaque areas and collagen. Briefly, the sections were stained with alcian blue, Verheoff's hematoxylin solution and then differentiated in $2 \%$ aqueous ferric chloride and subsequently stained in crocein scarlet-acid fuchsin and again differentiated in $5 \%$ aqueous phosphotungstic acid to achieve movat staining. The slides were stained with saturated picric acid Sirius red and alcoholic hematoxylin progressively, to observe lesional collagen content. All the stained slides were checked and captured microscopically by an upright microscope (Carl Zeiss AG, Oberkochen, Germany). Atherosclerotic lesions were analyzed using Image-Pro ${ }^{\circledR}$ Plus-6 software (Media Cybernetics, Inc., Rockville, MD, USA).

Immunohistochemistry. Regular sections from the excised aortic root were used for immunohistochemistry, to identify macrophages and smooth muscle cells (SMCs), according to a previously reported method (12). Briefly, sections were incubated with polyclonal antibodies at $37^{\circ} \mathrm{C}$ for $90 \mathrm{~min}$ or at $4^{\circ} \mathrm{C}$ overnight, and then labeled with horseradish peroxidase- or tetramethylrhodamine-conjugated anti-rabbit immunoglobulin G (catalog no. PV9000; OriGene Technologies, Inc., Beijing, China) at $37^{\circ} \mathrm{C}$ for $60 \mathrm{~min}$. The coverslips were subsequently mounted with $\mathrm{DABCO}^{\mathrm{TM}}$ mounting medium, and analyzed using an upright microscope (Carl Zeiss AG, Oberkochen, Germany). Anti- $\alpha$-actin (catalog no. sc-130616; 1:100) and anti-MAC-3, a highly glycosylated protein and a constituent of the lysosomal membrane that may be used as a macrophage marker, (or LAMP-2, catalog no. sc-81729; 1:100) antibodies were purchased from Santa Cruz Biotechnology, Inc. Dallas, TX, USA.

En face analysis of the descending aorta. The descending aortas were used for en face lipid staining. The aortas were dissected from the left subclavian artery to the iliac bifurcation, then opened longitudinally and stained with Oil Red O to visualize the extent of the lipid deposition. Aortic images were captured with a Sony DXC-960MD (Sony Corporation, Tokyo, Japan), and the lesion size was quantitatively analyzed. Data were analyzed with Image-Pro ${ }^{\circledR}$ Plus-6 software (Media Cybernetics, Inc.).

Cell culture and adhesion assays. CRL-1730 human umbilical vein vascular endothelial cells (HUVECs) were purchased from the American Type Culture Collection (Manassas, VA, USA) and were cultured in endothelial cell basal medium-2 Bullet kit media (Clonetics ${ }^{\mathrm{TM}}$; Lonza, Basel, Switzerland). Cells were grown to confluence at $37^{\circ} \mathrm{C}$ in $5 \%$ $\mathrm{CO}_{2}$, on $0.2 \%$ gelatin-coated culture dishes. Human monocytoid U937 cells (American Type Culture Collection) were cultured in RPMI-1640 medium supplemented with $10 \%$ fetal bovine serum (catalog no. 12633-012 and 10082139; Gibco; Thermo Fisher Scientific Inc., Waltham, MA, USA). To assess monocyte adhesion, HUVECs were plated onto 6-well gelatin-coated dishes at a density of $1.2 \times 10^{5}$ cells/well. The following day, cells were pretreated with oxidized-LDL (ox-LDL, catalog no. YB-002; Yiyuan Biotechnology, Guangzhou, China), after which MCP was added at various 
A

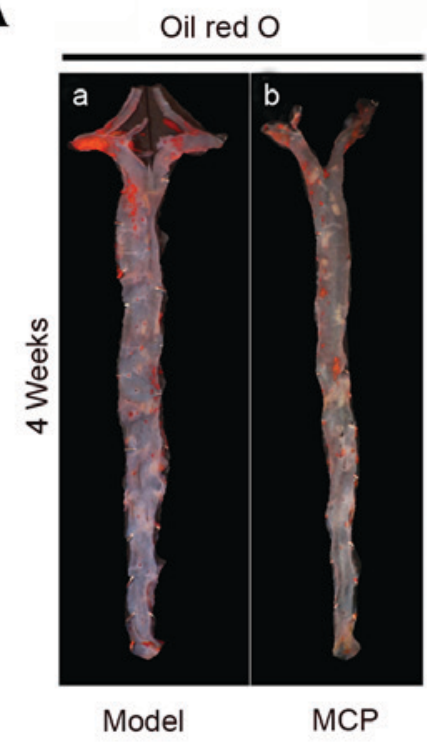

Aortic surface lesion area

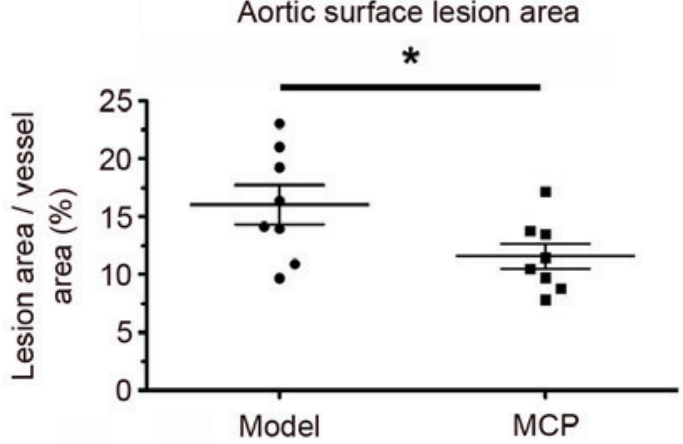

B
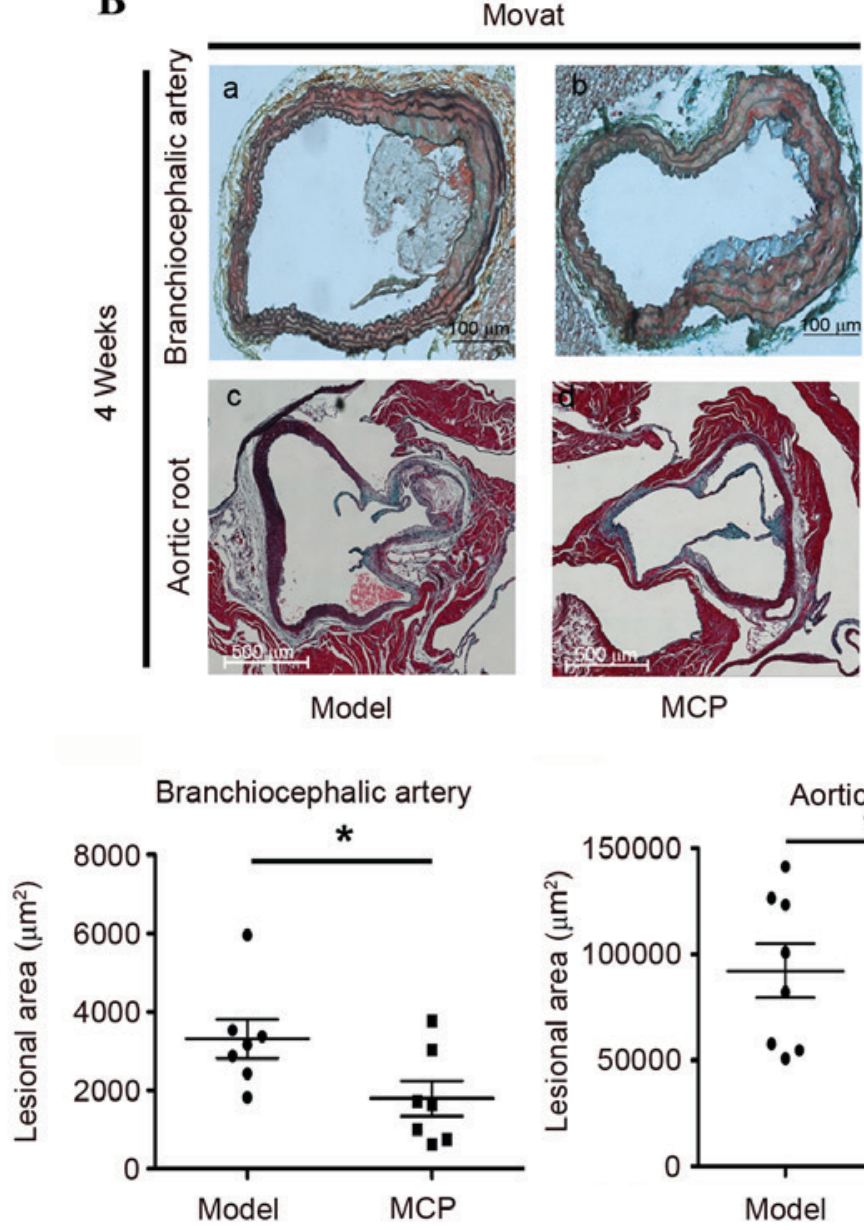

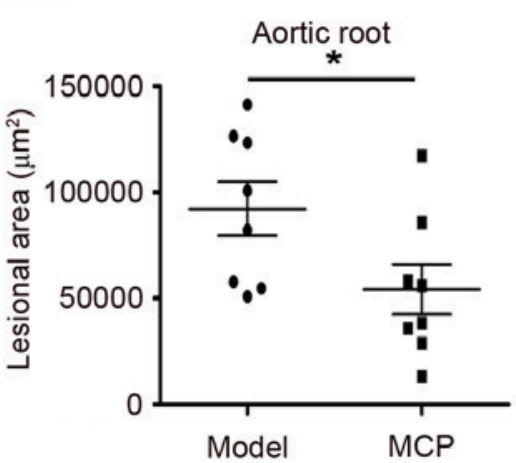

Figure 1. ApoE $\mathrm{E}^{-/-}$mice administered with MCP display reduced atherosclerotic lesion area. Eight-week-old male apoE ${ }^{-/-}$mice with a C57BL/6J background received an atherogenic diet, supplemented with MCP (1\%) in their drinking water for 4 weeks. (A) Relative size (\%) of the aortic atheroma lesion areas was determined by quantitative histomorphology of Oil Red O-stained en face specimens, for (a) model and (b) MCP-treated mice (n=8). (B) Lesion size in the branchiocephalic artery $(n=7)$ and aortic root $(n=8)$ for $(a$ and $c)$ model and (b and d) MCP-treated mice was determined by Movat staining. Data are presented as the mean \pm standard error of the mean. ${ }^{*} \mathrm{P}<0.05$ vs. the model group. $\mathrm{MCP}$, modified citrus pectin; apoE, apolipoprotein $\mathrm{E}$.

concentrations $(0.025,0.05,0.1$ and $0.25 \%$, for $24 \mathrm{~h})$. U937 cells $\left(3 \times 10^{5}\right.$ cells/well) were subsequently plated onto each monolayer, and incubated for $10 \mathrm{~min}$ under rotating conditions $(63 \mathrm{rpm})$, at room temperature. Non-adherent cells were removed by gentle washing with MCDB 131 medium (Invitrogen; Thermo Fisher Scientific, Inc., Waltham, MA, USA), and monolayers were fixed with $1 \%$ paraformaldehyde and observed with an inverted microscope (CKX41; Olympus Corporation, Tokyo, Japan).

Scanning electron microscopy (SEM). Branchiocephalic arteries (BCA) recovered from the mice were fixed with $2.5 \%$ gluteraldehyde in Millonig's phosphate buffer for $48 \mathrm{~h}$, followed by post-fixation with $1 \%$ osmium tetroxide for $45 \mathrm{~min}$. The specimens were dehydrated using increasing grades of ethyl alcohol (15 min in 50, 70, 80, and 95\% alcohol, followed by three 10 -min periods in $100 \%$ alcohol) and subsequently placed in an LPD-100 critical point dryer (S4800; Hitachi Limited, Inc., Tokyo, Japan) for $5 \mathrm{~min}$ at $41^{\circ} \mathrm{C}$ and $1,200 \mathrm{psi}$ $\mathrm{CO}_{2}$. The tissues were then mounted on aluminum stubs with silver glue, sputter coated with gold, and examined using an SEM $(17,18)$.
Statistical analysis. All values were presented as the mean \pm standard error of the indicated number of measurements. An unpaired Student's t-test and analysis of variance were conducted, followed by post hoc testing with the Tukey procedure to determine significance. $\mathrm{P}<0.05$ was considered to indicate a statistically significant difference.

\section{Results}

MCP treatment reduces the size of atherosclerotic lesions in $a p o E^{-/}$mice. MCP is a naturally occurring inhibitor of galectin-3 carbohydrate binding $(1,2)$. To investigate whether in vivo galectin-3 inhibition could reduce plaque size, apoE ${ }^{-/-}$mice were fed an atherogenic diet, with MCP (1\%) supplemented in their drinking water, for 4 weeks. The size of the aortic lesion vs. the total arch area was determined, via quantitative histomorphological analysis of Oil Red $\mathrm{O}$-stained en face specimens of the descending aorta. The area of lesioned aorta, compared with the total arch area, was reduced following MCP administration for 4 weeks (Fig. 1A). Similarly, the lesion size on the BCA and the aortic root was reduced in the MCP group, compared with the model group, as 

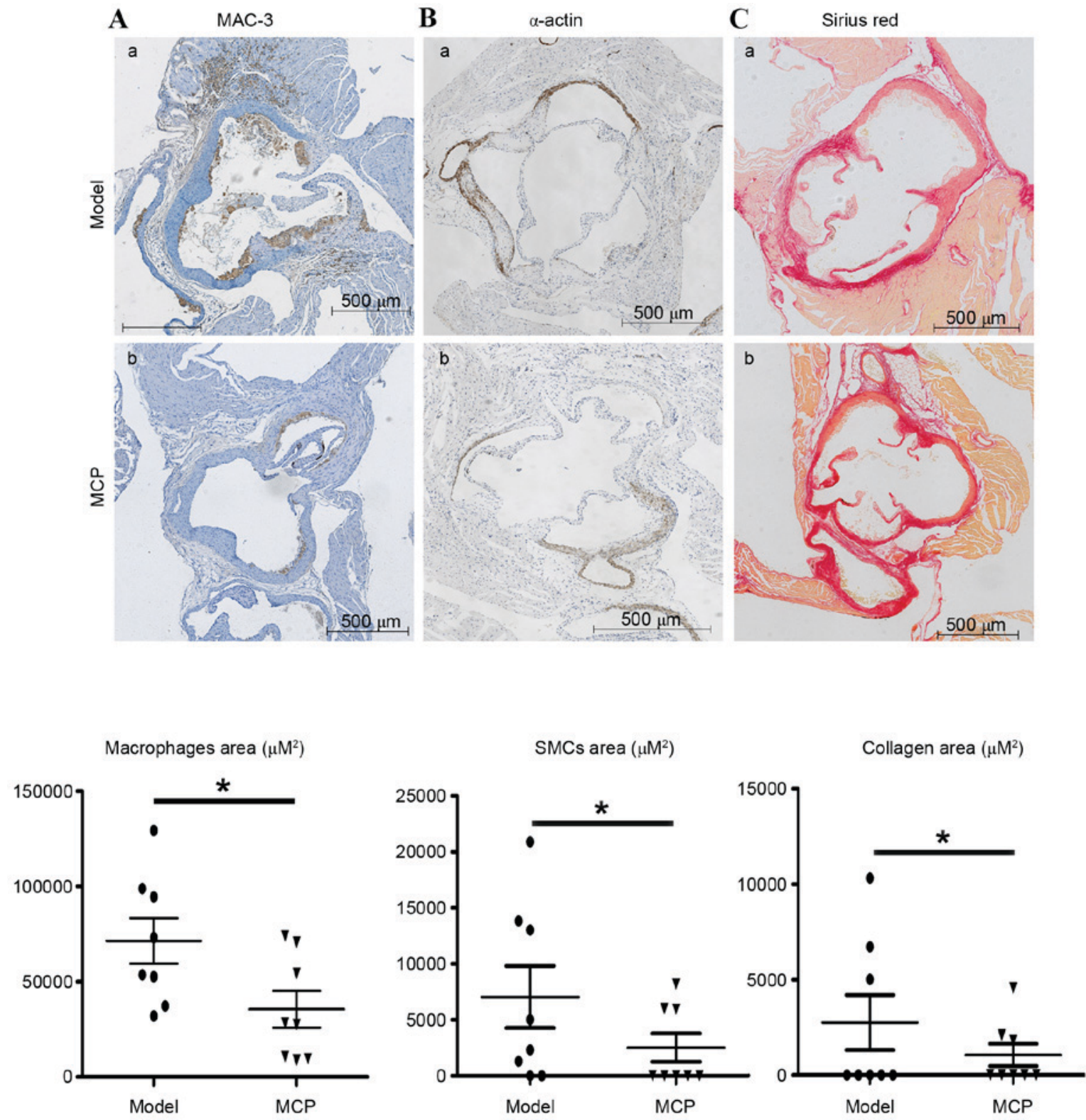

Figure 2. MCP alters plaque structure and composition in apolipoprotein E-deficient mice. Atherosclerotic lesions are mainly composed of macrophages, SMCs and collagen. Immunohistochemical analysis of aortic root plaques revealed decreased (A) MAC-3, a marker of macrophages ( $\mathrm{n}=8$ ), (B) SMC $\alpha$-actin $(\mathrm{n}=8)$ and $(\mathrm{C})$ collagen $(\mathrm{n}=8)$, in apoE $\mathrm{E}^{-/}$mice administered with $(\mathrm{A}-\mathrm{b}, \mathrm{B}-\mathrm{b}$ and $\mathrm{C}-\mathrm{b}) \mathrm{MCP}$, compared with the (A-a, B-a and C-a) model group. Data are presented as the mean \pm standard error of the mean. ${ }^{*} \mathrm{P}<0.05$ vs. model group. MCP, modified citrus pectin; SMCs, smooth muscle cells.

evidenced by Movat staining (Fig. 1B). These results indicated that MCP may reduce the size of the atherosclerotic lesion in $\mathrm{apoE}^{-/-}$mice.

MCP alters plaque structure and composition in apo $E^{-1-}$ mice. Atherosclerotic lesions are predominantly composed of macrophages, SMCs and collagen. Immunohistochemical probing for MAC-3, a marker of macrophages, indicated that MCP treatment resulted in fewer macrophages in the atherosclerotic lesions (Fig. 2A). Similarly, the amount of collagen and the number of SMCs were also reduced in the MCP-treated aortic root plaques (Fig. 2B and C).

MCP reduces in vitro adhesion of U937 monocytes to HUVECs. HUVECs were pretreated with ox-LDL and subsequently co-cultured with U937 cells. Treatment of HUVECs with
ox-LDL increased the number of adherent monocytes (Fig. 3). However, subsequent incubation with various concentrations of $\operatorname{MCP}(0.025,0.05,0.1$ and $0.25 \%)$ reduced the number of adherent U937 monocytes (Fig. 3).

MCP impairs endothelial injury in apoE $E^{-1}$ mice. SEM analysis was performed on the surface of the atheroma-prone vessel wall in apoE $\mathrm{E}^{-/-}$mice. Pathological alterations caused by endothelial injury were observed in the model group; adherent monocytes were located at breakages in the endothelial cell junction (Fig. 4A-a). In the MCP group, endothelial junction sites were loose, however they did not appear to be broken (Fig. 4B-a). Notably, a monocyte was observed entering the endothelial barrier (Fig. 4B-b). From these results, it was speculated that the endothelial cell junctions recombined loosely, following monocyte transmigration, in 
A

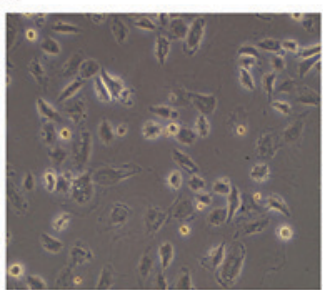

Control

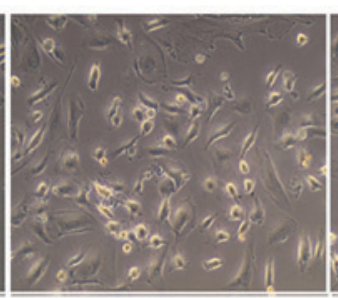

ox-LDL

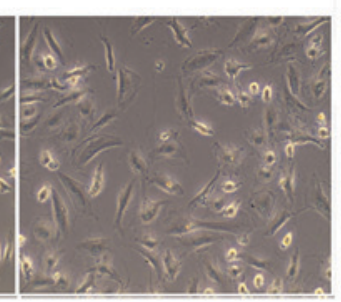

ox-LDL+0.025\% MCP

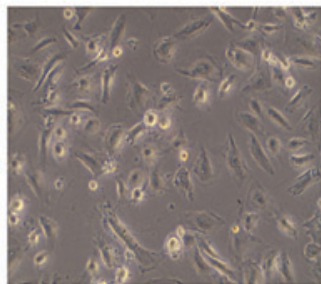

ox-LDL+0.05\% MCP

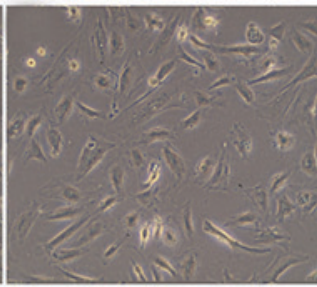

ox-LDL+0.1\% MCP

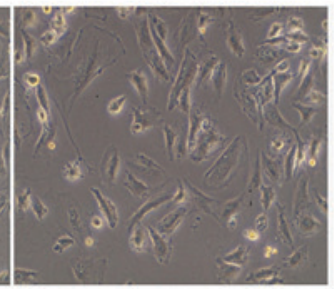

ox-LDL+0.25\% MCP

\section{B}

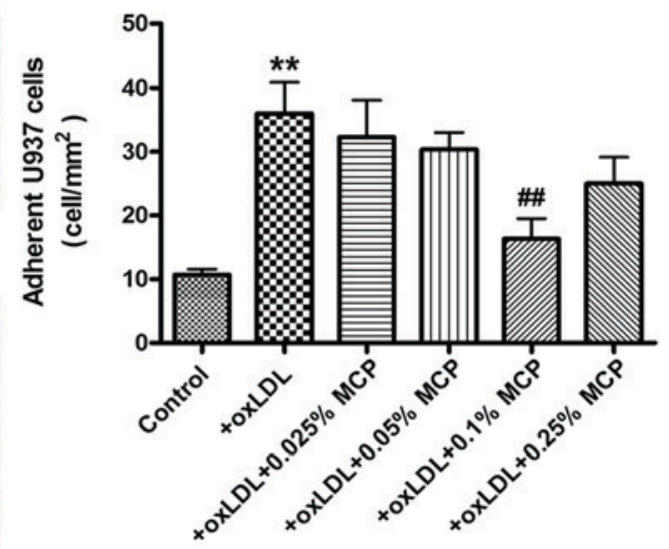

Figure 3. MCP reduces the adhesion of U937 monocytes to HUVECs in vitro. HUVECs pretreated with ox-LDL were co-cultured with U937 cells. Treatment of HUVECs with ox-LDL increased the number of adherent monocytes. Incubation with various concentrations of MCP $(0.025,0.05,0.1$ and $0.25 \%)$ reduced the number of adherent monocytes (A) Cell images and (B) histogram analysis of adherent monocytes. Data are presented as the mean \pm standard error of the mean. ${ }^{* *} \mathrm{P}<0.01$ vs. control group; ${ }^{\# \#} \mathrm{P}<0.01$ vs. ox-LDL group. Magnification, $\mathrm{x} 100$. MCP, modified citrus pectin; ox-LDL, oxidized-low density lipoprotein; HUVECs, human umbilical vein endothelial cells.

A

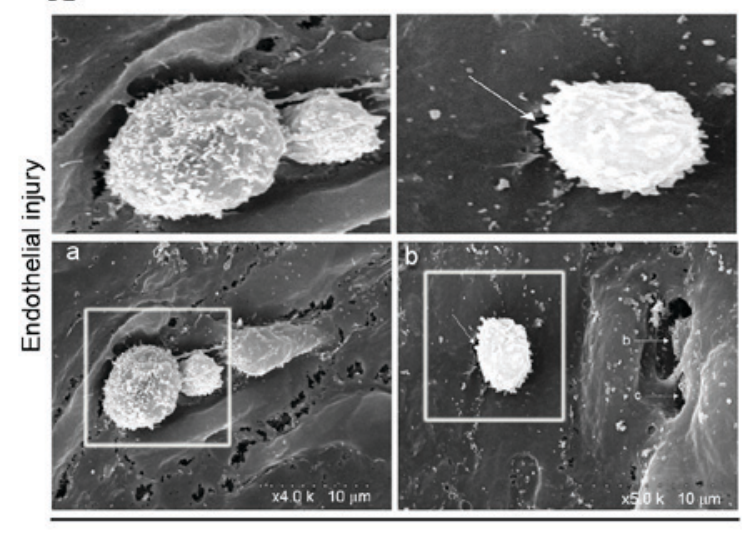

Model

C

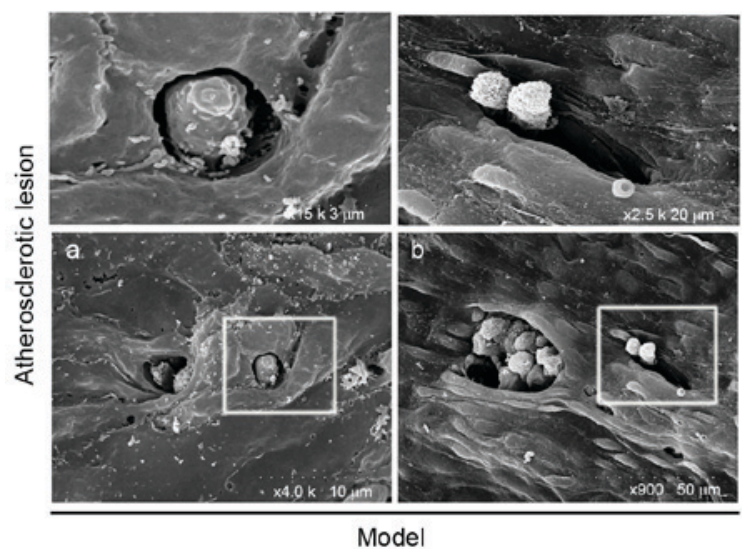

B

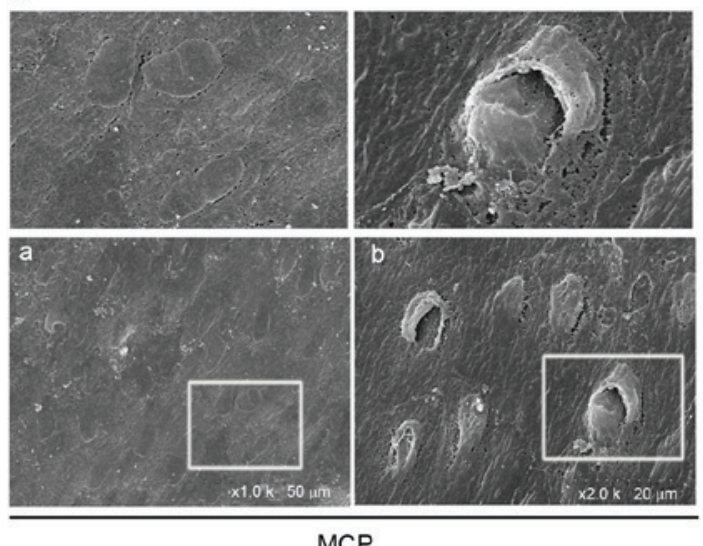

D

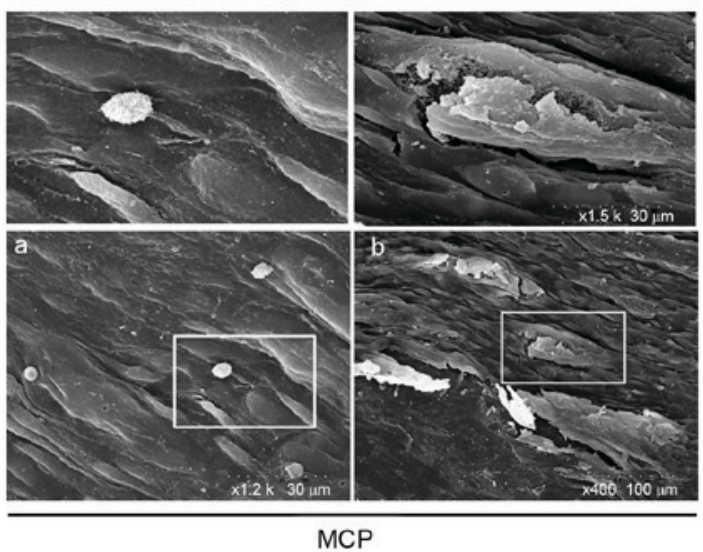

Figure 4. MCP impairs endothelial injury in apoE ${ }^{-/}$mice. Analysis of the surface of the atheroma-prone vessel wall in apoE ${ }^{-/}$mice was performed using scanning electron microscopy. Enlarged images of the lower panels are displayed in the upper panels. Endothelial injury was imaged in (A) model and (B) MCP-treated mice. (A-a and -b) Adherent monocytes were located at the break of the endothelial joint, whereas (B-a and -b) endothelial junction sites were only loosened in the MCP group. (B-b) A monocyte can also be seen entering the endothelial barrier. The atherosclerotic lesion in (C) the model group had several disruption holes or 'craters' indicated in C-a and b, compared with the MCP-treated group with complete surfaces present in (D-a) aortic vessels and D-b atherosclerotic lesions. MCP, modified citrus pectin; apoE, apolipoprotein E. 
the MCP-treated group. Furthermore, the lesion surface in the model group (Fig. 4C-a and -b) contained multiple disruption holes or 'craters', whereas the endothelium of the MCP group appeared to be of greater integrity (Fig. 4D-a and -b).

\section{Discussion}

The present study demonstrated that MCP-treated apoE $\mathrm{E}^{-1-}$ mice developed smaller atherosclerotic lesions, accompanied by fewer macrophages and SMCs and reduced amounts of collagen. Previous research (13) indicated that galectin-3-deficient mice had smaller atherosclerotic plaque volumes, and reduced intralesional macrophage accumulation and activation. Furthermore, the orally active inhibitor of galectin-3, $\mathrm{MCP}$, may also reduce the atherosclerotic area; however, the precise mechanism remains unclear. The present study was therefore conducted to further investigate the role of galectin-3 in atherosclerosis. $\mathrm{ApoE}^{-/-}$mice fed an atherogenic diet $(1.25 \%$ cholesterol) for 4 weeks displayed early or initial lesions (14); $1 \%$ MCP was therefore supplemented into the drinking water for 4 weeks, to evaluate the effects of MCP in early plaque formation. Oil Red $\mathrm{O}$ staining of en face specimens was performed to evaluate the total atherosclerotic area, and this revealed reduced aortic lesion areas in the MCP group, compared with the model group. Furthermore, the BCA and aortic root were examined pathologically, and lesional areas were observed to be significantly smaller in the MCP group compared with the model group.

Macrophages are the predominant cell type present in early atheroma plaques; they release various proinflammatory cytokines and chemokines, thereby inducing additional circulating monocytes to gather at the atheroma-prone areas, and to further differentiate into lesional macrophages, which is followed by vascular medial SMC migration and collagen synthesis (3). Analysis of the composition of the MCP-treated group lesions indicated fewer macrophages and SMCs, and reduced amounts of collagen, compared with the model group, thereby indicating that MCP may be able to protect against atherosclerotic development. Macrophages may interact with the ECM, at least partly, via galectin-3-induced differentiation and maturation (15). Galectin-3 is also chemotactic, guiding macrophages to lectin-rich locations in vivo and in vitro, and, similarly to monocyte chemoattractant protein-1, it may induce $\mathrm{Ca}^{2+}$ influx in monocytes; the chemotactic effect and the induction of $\mathrm{Ca}^{2+}$ influx may involve a PTX-sensitive pathway (10). Furthermore, galectin-3 is present on macrophages in atherosclerotic areas, and increased extracellular galectin-3 expression and secretion is a feature of alternative macrophage activation; galectin-3 interaction with CD98 results in the activation of phosphoinositide- 3 kinase, and thus the alternative activation of macrophages. In addition, the interleukin-4-induced alternative activation pathway may be blocked via a specific inhibitor of extracellular galectin-3 carbohydrate binding (16). These results indicated that a galectin-3 feedback loop may induce alternative macrophage activation. Pharmacological modulation of galectin-3 function may therefore represent a novel therapeutic strategy in alternatively activated macrophage pathologies (19). The present study detected significantly reduced macrophage- and SMC-positive areas in atherosclerotic plaques in MCP-treated apoE ${ }^{-/-}$mice. Galectin-3 may promote the expression of monocytic chemokines, causing monocytes to preferentially migrate to, and accumulate in, vascular endothelial and arterial plaque lesions, particularly in lipid cores. This triggers the adhesion of macrophages to vascular endothelial cells, via interaction with laminin, fibronectin, elastin and collagen IV fibers (8). The transmigrated macrophages communicate with SMCs, leading to SMC activation and proliferation, in turn resulting in the appearance of SMCs in the atherosclerotic plaque. Since SMCs also synthesize collagen and other ECM components present in atherosclerotic lesions, a reduction in SMCs observed in the MCP group was concurrent with decreased amounts of collagen in the same group. Since lesional macrophages trigger and expand the accumulation of additional macrophages and medial SMCs to the lesion, the anti-atheroma effects of MCP may therefore mainly result from its inhibitory impact on macrophage adhesion.

The role served by the interaction between endothelial cells and monocytes/macrophages in atherosclerotic initiation is well documented (1). Monocyte and macrophage adhesion and sub-endothelial migration are the predominant processes associated with atherosclerotic initiation. Atheroma-prone areas are inflammatory sites; these may appeal to monocytes leaving the circulation, thus leading to endothelial cell anchorage via sequential attachments, which are mediated by several adhesion molecules, including galectin-3. It has previously been indicated that galectin-3 is expressed by various cell types including endothelial, epithelial and dendritic cells, and numerous inflammatory cells such as monocytes/macrophages, mast cells, neutrophils and eosinophils (20). Furthermore, galectin-3 is distributed on the cell surface, cytoplasm, and nucleus, as well as in the extracellular environment, indicating the multi-functionality of this lectin (21). Galectin-3 enables cell adhesion via cell-ECM-protein and cell-cell interactions. ECM proteins include laminin, fibronectin, proteoglycans, collagen and vitronectin, which are distributed in endothelial cells, the interstitial space and the basement membrane, and serve important roles in cell adherence (22). Galectin-3 participates in the ECM-cell adhesion process, and the association between galectin-3 and ECM proteins, particularly laminin and fibronectin, is essential for leucocyte movement. Furthermore, the interaction of galectin-3 with fibronectin is unique for the macrophage migratory process (23), indicating that galectin-3 may guide monocyte-derived macrophages to transmigrate across the endothelium and reach the arterial media, via its ECM binding ability. Previous studies have demonstrated that galectin-3 has no impact on the expression of adhesion molecules, such as selectin, ICAM, VCAM and very late antigen-4, indicating that galectin-3 is not an inflammatory modulator. Therefore, galectin-3 may predominantly guide macrophage movement via its binding affinity with the redistributed ligands in various vascular tissues. The associations between leucocytes and endothelial cells are primarily mediated by the interaction of various adhesion molecules, such as selectins and VCAM-1 to $\alpha 4 \beta 1$ integrin, and ICAM- 1 to $\beta 2$-integrin (24). VCAM-1 may mediate rolling-type adhesion and firm adhesion, depending on the avidity status of $\alpha 4 \beta 1$-integrin (25). It has been demonstrated that galectin-3 is capable of associating with $\beta 1$ integrin, via its carbohydrate recognition domain, in a lactose-dependent manner (23). The binding of galectin-3 with $\alpha 4 \beta 1$ integrin may further modulate its receptor state and alter 
its affinity to VCAM-1; therefore, galectin-3 may be indirectly involved in $\alpha 4 \beta 1$-integrin-VCAM-1 dependent rolling and adhesion. To identify the impact of galectin-3 on endothelial cell-monocyte adhesion, the present study treated U937 monocytes with MCP, followed by co-incubation with ox-LDL stimulated HUVECs. The results revealed significantly greater numbers of adherent U937 monocytes on ox-LDL stimulated HUVECs, compared with controls. However, the number of adherent U937 cells was markedly reduced when they were pretreated with only $0.1 \% \mathrm{MCP}$.

The transendothelial migration of monocytes requires dynamic morphological alterations in endothelial cells. Monocyte adhesion induces endothelial F-actin contraction, thereby generating tension and facilitating the necessary changes to cellular morphology, which results in endothelial deformability (26). SEM analysis of transendothelial macrophages revealed large endothelial structure disruptions in the model group, whereas MCP-treated tissues had only a loosened endothelial structure. Furthermore, endothelial cell destruction was frequent in the atherosclerotic surfaces of the model group, whereas the atherosclerotic plaque surface remained intact in the MCP-treated group.

In conclusion, the present study demonstrated that MCP, a galectin-3 inhibitor, reduced atherosclerotic lesions via the inhibition of leucocyte adhesion to endothelial cells. Inhibiting galectin-3 function may be a promising therapeutic strategy for the treatment of atherosclerosis.

\section{Acknowledgements}

This study was supported by funding from the National Basic Research Program of China (grant no. 2012CB517502) and the National Natural Science Foundation of China (grant nos. 81270887 and 81070634).

\section{References}

1. Chistiakov DA, Revin VV, Sobenin IA, Orekhov AN and Bobryshev YV: Vascular endothelium: Functioning in norm, changes in atherosclerosis and current dietary approaches to improve endothelial function. Mini Rev Med Chem 15: 338-350, 2015.

2. Schäfer A and Bauersachs J: Endothelial dysfunction, impaired endogenous platelet inhibition and platelet activation in diabetes and atherosclerosis. Curr Vasc Pharmacol 6: 52-60, 2008.

3. Blankenberg S, Barbaux S and Tiret L: Adhesion molecules and atherosclerosis. Atherosclerosis 170: 191-203, 2003.

4. Rao SP, Wang Z, Zuberi RI, Sikora L, Bahaie NS, Zuraw BL, Liu FT and Sriramarao P: Galectin-3 functions as an adhesion molecule to support eosinophil rolling and adhesion under conditions of flow. J Immunol 179: 7800-7877, 2007.

5. Soehnlein O: Multiple roles for neutrophils in atherosclerosis. Circ Res 110: 875-888, 2012.

6. Nieminen J, St-Pierre C, Bhaumik P, Poirier F and Sato S: Role of galectin-3 in leukocyte recruitment in a murine model of lung infection by Streptococcus pneumoniae. J Immunol 180: 2466-2473, 2008.
7. Tadokoro T, Ikekita M, Toda T, Ito H, Sato T, Nakatani R, Hamaguchi Y and Furukawa K: Involvement of Galectin-3 with vascular cell adhesion molecule-1 in growth regulation of mouse BALB/3T3 cells. J Biol Chem 284: 35556-35563, 2009.

8. Inohara H, Akahani S, Koths K and Raz A: Interactions between galectin-3 and Mac-2-binding protein mediate cell-cell adhesion. Cancer Res 56: 4530-4534, 1996.

9. Sasaki T, Brakebusch C, Engel J and Timpl R: Mac-2 binding protein is a cell-adhesive protein of the extracellular matrix which self-assembles into ring-like structures and binds betal integrins, collagens and fibronectin. EMBO J 17: 1606-1613, 1998.

10. Sano H, Hsu DK, Yu L, Apgar JR, Kuwabara I, Yamanaka T, Hirashima M and Liu FT: Human galectin-3 is a novel chemoattractant for monocytes and macrophages. J Immunol 165: 2156-2164, 2000.

11. Hopkins PN: Molecular biology of atherosclerosis. Physiol Rev 93: 1317-1542, 2013.

12. Tekabe Y, Li Q, Rosario R, Sedlar M, Majewski S, Hudson BI, Einstein AJ, Schmidt AM and Johnson LL: Development of receptor for advanced glycation end products-directed imaging of atherosclerotic plaque in a murine model of spontaneous atherosclerosis. Circ Cardiovasc Imaging 1: 212-219, 2008.

13. Gao X, Zhi Y, Zhang T, Xue H, Wang X, Foday AD, Tai G and Zhou Y: Analysis of the neutral polysaccharide fraction of MCP and its inhibitory activity on galectin-3. Glycoconj J 29: 159-165, 2012.

14. Nachtigal M, Ghaffar A and Mayer EP: Galectin-3 gene inactivation reduces atherosclerotic lesions and adventitial inflammation in ApoE-deficient mice. Am J Pathol 172: 247-255, 2008.

15. Jacob SS, Shastry P and Sudhakaran PR: Monocyte-macrophage differentiation in vitro: Modulation by extracellular matrix protein substratum. Mol Cell Biochem 233: 9-17, 2002.

16. MacKinnon AC, Farnworth SL, Hodkinson PS, Henderson NC, Atkinson KM, Leffler H, Nilsson UJ, Haslett C, Forbes SJ and Sethi T: Regulation of alternative macrophage activation by galectin-3. J Immunol 180: 2650-2658, 2008.

17. Walski M, Chlopicki S, Celary-Walska R and FrontczakBaniewicz M: Ultrastructural alterations of endothelium covering advanced atherosclerotic plaque in human carotid artery visualized by scanning electron microscope. J Physiol Pharmacol 53: 713-723, 2002.

18. Nathan L, Pervin S, Singh R, Rosenfeld M and Chaudhuri G: Estradiol inhibits leukocyte adhesion and transendothelial migration in rabbits in vivo: Possible mechanisms for gender differences in atherosclerosis. Circ Res 85: 377-385, 1999.

19. MacKinnon AC, Liu X, Hadoke PW, Miller MR, Newby DE and Sethi T: Inhibition of galectin-3 reduces atherosclerosis in apolipoprotein E-deficient mice. Glycobiology 23: 654-663, 2013.

20. Swarte VV, Mebius RE, Joziasse DH, Van den Eijnden DH and Kraal G: Lymphocyte triggering via L-selectin leads to enhanced galectin-3-mediated binding to dendritic cells. Eur J Immunol 28: 2864-2871, 1998.

21. Funasaka T, Raz A and Nangia-Makker P: Galectin-3 in angiogenesis and metastasis. Glycobiology 24: 886-891, 2014.

22. Hynes RO: The extracellular matrix: Not just pretty fibrils. Science 326: 1216-1219, 2009.

23. Hashiba K, Sano M, Nio-Kobayashi J, Hojo T, Skarzynski DJ and Okuda K: Galectin-3 contributes to luteolysis by binding to Beta 1 integrin in the bovine corpus luteum. Biol Reprod 91: 2, 2014.

24. Hatley ME, Srinivasan S, Reilly KB, Bolick DT and Hedrick CC: Increased production of 12/15 lipoxygenase eicosanoids accelerates monocyte/endothelial interactions in diabetic $\mathrm{db} / \mathrm{db}$ mice. J Biol Chem 278: 25369-25375, 2003.

25. Cybulsky MI, Iiyama $\mathrm{K}$, Li $\mathrm{H}$, Zhu S, Chen M, Iiyama M, Davis V, Gutierrez-Ramos JC, Connelly PW and Milstone DS: A major role for VCAM-1, but not ICAM-1, in early atherosclerosis. J Clin Invest 107: 1255-1262, 2001.

26. Ingber DE: Cellular tensegrity: Defining new rules of biological design that govern the cytoskeleton. J Cell Sci 104: 613-627, 1993. 